\title{
Use of Michelson and Fabry-Perot interferometry for independent determination of the refractive index and physical thickness of wafers
}

\author{
Glen D. Gillen and Shekhar Guha
}

\begin{abstract}
We present a method to independently measure the refractive index and the thickness of materials having flat and parallel sides by using a combination of Michelson and Fabry-Perot interferometry techniques. The method has been used to determine refractive-index values in the infrared with uncertainties in the third decimal place and thicknesses accurate to within $\pm 5 \mu \mathrm{m}$ for materials at room and cryogenic temperatures. (C) 2005 Optical Society of America
\end{abstract}

OCIS codes: $120.2230,120.3180,120.4290,160.4760$.

The refractive index, $n$, and the thermo-optic coefficient, $\mathrm{d} n / \mathrm{d} T$, of materials are often determined by one's interferometrically measuring the phase change that light undergoes in passing through a plane-parallel slab of the material. Because the phase change depends on the value of $n$ as well as the slab thickness, $d$, to obtain accurate values of $n$ and $\mathrm{d} n / \mathrm{d} T$, it is important to know $d$ accurately. FabryPerot etalon interferometry has been used to optically measure $d,{ }^{5}$ but the precision of thickness measurements with this method is limited by the precision of the known refractive-index value. Recent research by Coppala et al. ${ }^{6}$ demonstrated that independent values for $n$ and $d$ can be obtained with interferometry and a continuously tunable laser source.

In this paper we demonstrate that the Michelson and the Fabry-Perot interferometric methods can be used sequentially to determine independent and absolute values of both the material's thickness and the material's refractive index over a wide range of temperatures of practical interest. The method does not require that either quantity be initially well known. With this method, both $n$ and $d$ can be determined by

G. D. Gillen (ggillen@anteon.com) is with Air Force Research Laboratory, Materials and Manufacturing Directorate, Anteon Corporation, Wright-Patterson Air Force Base, Dayton, Ohio. S. Guha is with Air Force Research Laboratory, Materials and Manufacturing Directorate, Wright-Patterson Air Force Base, Dayton, Ohio.

Received 6 May 2004; revised manuscript received 24 September 2004; accepted 13 October 2004.

0003-6935/05/030344-04\$15.00/0

(C) 2005 Optical Society of America use of a fixed-wavelength laser source. First, by use of data from both experiments, the material's physical thickness is determined. Then the thickness value is used to determine the material's refractive index (and thermo-optic coefficient) with either of the interferometric methods. We present experimental verification of this method by measuring $n$ and $d$ for a range of common infrared materials at both room temperature and cryogenic temperatures.

The intensity of a coherent collimated beam of light transmitted by a plane-parallel transparent plate is given by the Airy formula ${ }^{7}$ :

$$
I_{t}=\left[\frac{\left(1-r^{2}\right)^{2}}{1+r^{4}-2 r^{2} \cos \phi_{f}}\right] I_{o},
$$

where $I_{o}$ is the incident intensity, $r$ is the reflection coefficient for the electric field, and $\phi_{f}$ is the phase difference accumulated by the light beam in a double traversal through the plate.

As the sample is rotated in the path of the laser light, the net transmitted intensity will modulate owing to the changing phase, $\phi_{f}$. The angle-dependent phase difference between subsequent transmitted light paths through the sample is given by ${ }^{7}$

$$
\phi_{f}(\theta)=\frac{4 \pi n d}{\lambda} \cos \theta_{t}=\frac{4 \pi d}{\lambda} \sqrt{n^{2}-\sin ^{2} \theta},
$$

where $d$ is the sample thickness, $\lambda$ is the laser wavelength, $\theta_{t}$ is the angle of refraction, and $\theta$ is the angle of incidence of the laser path with respect to the normal of the sample surface. 


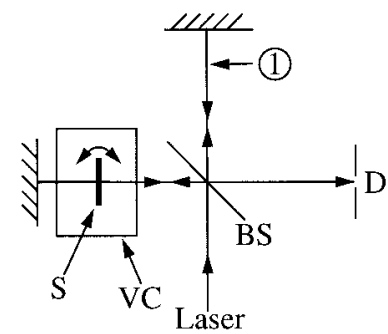

Fig. 1. Experimental setup for Michelson interferometry and Fabry-Perot etalon interferometry, where $\mathrm{S}$ is the sample, $\mathrm{VC}$ is the vacuum chamber, BS is the beam splitter, and D is the detector. For Fabry-Perot etalon experiments, the reference beam is blocked at position 1 .

If a flat-parallel plate is inserted into one arm of a Michelson interferometer and rotated about an axis perpendicular to the laser beam propagation path, the observed intensity exiting the interferometer is given by ${ }^{8}$

$$
I=I_{r}+I_{s}+2 \sqrt{I_{r} I_{2}} \cos \phi_{m}
$$

where $I_{r}$ and $I_{s}$ are the intensities incident on the detector due to the reference arm and the sample arm of the interferometer, respectively. The angledependent phase term, $\phi_{m}$, is

$$
\phi_{m}(\theta)=\left[\frac{4 \pi d}{\lambda}\left(\sqrt{n^{2}-\sin ^{2} \theta}+1-\cos \theta\right) .\right.
$$

The phase information for either the Fabry-Perot etalon interferometer or the Michelson interferometer can be extracted as a function of the sample plate's angle by observation of the intensity modulations. Subtracting Eq. (2) from Eq. (4) yields the difference between the two phases, or

$$
\phi_{m}(\theta)-\phi_{f}(\theta)=\frac{4 \pi d}{\lambda}(1-\cos \theta)
$$

which is independent of the refractive index of the sample plate medium. The material's physical thickness is now decoupled from the material's refractive index. Thus, with the phase-difference information, the absolute thickness of the sample plate can be determined. Then, with the known thickness, the absolute refractive index can be determined by either interferometric technique.

Figure 1 shows the experimental setup for sequential Fabry-Perot etalon and Michelson interferometric data runs. The experimental setup is essentially the same as previously described. ${ }^{8}$ The laser source is a grating-tuned carbon dioxide laser from Access Laser with an output of approximately $400 \mathrm{~mW}$ at a wavelength of $10.611 \mu \mathrm{m}$. The incident light is $s$ polarized with respect to the sample surface. To perform measurements on cryogenic samples, we house

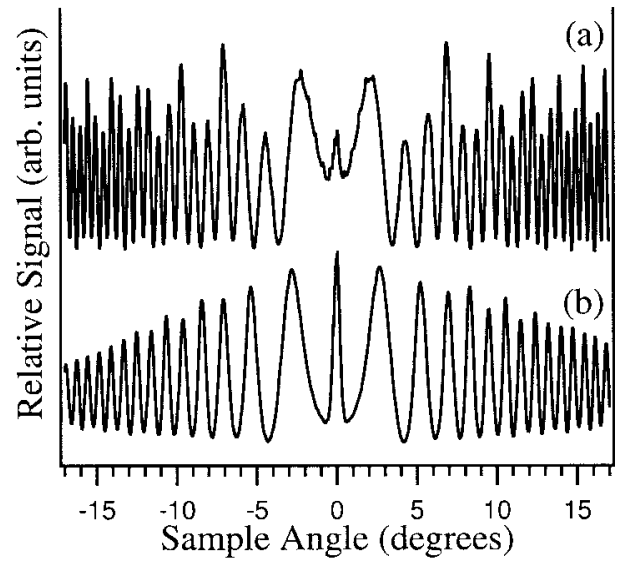

Fig. 2. Observed relative intensity fluctuations at the detector for (a) Michelson interferometry and (b) Fabry-Perot interferometry for a $\mathrm{ZnSe}$ window at $296 \mathrm{~K}$ and having a thickness of $4 \mathrm{~mm}$. The intensity spike at $0 \mathrm{deg}$ is due to the reflected beam at normal incidence.

the sample inside a homebuilt vacuum chamber and mounted it in thermal contact to the bottom of a liquid-nitrogen holding cell. The intensity at the detector is recorded as a function of the sample plate's angular position. For the Fabry-Perot etalon interferometric data run, the reference beam is blocked at position 1 in Fig. 1. Since the beam passes through the sample twice, Eq. (1) becomes

$$
I_{t}=\left[\frac{\left(1-r^{2}\right)^{2}}{1+r^{4}-2 r^{2} \cos \phi_{f}}\right]^{2} I_{o} .
$$

Although the observed intensity is different from a single-pass Fabry-Perot interferometer, the angular locations of the maxima and minima do not change.

Typical observed interference patterns as functions of the sample orientation are illustrated in Fig. 2. Figure 2(a) is the observed relative intensity at the detector for a Michelson interferometry setup, as a function of the sample angle. Figure 2(b) is the observed relative intensity at the detector for a FabryPerot etalon interferometry setup (an identical experimental setup except with the reference arm blocked.) The raw data displayed in Fig. 2 are for a ZnSe window, at $296 \mathrm{~K}$ and with a vendor-specified thickness of $4 \mathrm{~mm}$, exposed to laser light with a wavelength of $10.611 \mu \mathrm{m}$.

We extract the phase information from the raw data by determining the angular position for each maximum and minimum. Maxima observed for Michelson interferometry are assigned phase values of even multiples of $\pi$, and minima are assigned phase values of odd multiples of $\pi$, the integer values of which are determined by their observed fringe number. The phase values for $\phi_{f}$ are assigned negative multiple values of $\pi$ owing to the fact that Eq. (2) is a decreasing function with respect to increasing $\theta$. The 
Table 1. Temperature-Dependent Refractive-Index Results for GaAs, $\mathrm{Ge}, \mathrm{Si}$, and $\mathrm{ZnSe}$

\begin{tabular}{lccc}
\hline & Temperature & & $n$, Reported \\
Material & $(\mathrm{K})$ & $n$, Measured & or Predicted \\
\hline $\mathrm{GaAs}$ & 296 & $3.307 \pm 0.017$ & 3.2727 at $10.3 \mu \mathrm{m}$ \\
$\mathrm{Ge}$ & 296 & $4.0093 \pm 0.0010$ & 4.0049 at $10.6 \mu \mathrm{m}$ \\
& 93 & $3.9374 \pm 0.0028$ & 3.9216 at $10.6 \mu \mathrm{m}$ \\
$\mathrm{Si}$ & 296 & $3.4139 \pm 0.0024$ & 3.4152 at $10.6 \mu \mathrm{m}$ \\
$\mathrm{ZnSe}$ & 296 & $2.4056 \pm 0.0021$ & 2.4029 at $10.6 \mu \mathrm{m}$ \\
& 97 & $2.3947 \pm 0.0021$ & 2.3910 at $10.6 \mu \mathrm{m}$ \\
\hline
\end{tabular}

All measurements were performed for a wavelength of $10.611 \mu \mathrm{m}$, with initial guesses of $n=4$.

See Ref. 10

See Ref. 9.

extracted locations of the maxima and minima of the Michelson interferometry data are fit to the equation

$$
\begin{aligned}
\phi_{m}(\theta)= & m_{m}(\theta) \pi=\frac{4 \pi d_{g}}{\lambda}\left[\sqrt{n_{t}^{2}-\sin ^{2}\left(\theta-\theta_{o}\right)}\right. \\
& \left.+1-\cos \left(\theta-\theta_{o}\right)\right]+\phi_{o},
\end{aligned}
$$

where $m_{m}$ represents positive integer values, $d_{g}$ is the initial guess value for the thickness, $n_{t}$ is a temporary fit parameter for the refractive index, $\theta_{o}$ is a fit parameter for the angular offset, and $\phi_{o}$ is a fit parameter for the phase offset for $\theta=0$. Similarly, the raw data from Fabry-Perot etalon scans are fit to

$\phi_{f}(\theta)=m_{f}(\theta) \pi=\frac{4 \pi d_{g}}{\lambda} \sqrt{n_{t}^{2}-\sin ^{2}\left(\theta-\theta_{o}\right)}+\phi_{o}$,

where $m_{f}$ represents negative integer values. Fitting the experimental raw data to Eqs. (7) and (8) does not result in accurate numbers for either the thickness or the refractive index but rather accurate mathematical fits for $\phi_{m}(\theta)$ and $\phi_{f}(\theta)$ by use of the fitting routine variables $n_{t}, \theta_{o}$, and $\phi_{o}$. The physical thickness of the wafer, $d$, can then be determined with Eq. (5), and the values of the parameters can be determined by the two mathematical fits. Finally, the refractive index of the medium, $n$, can be determined by either Eq. (7) or (8) with this calculated value of $d$ and allowing $n_{t}$ to become a fitting routine variable.

Table 1 summarizes some of the experimental results obtained for gallium arsenide, germanium, silicon, and zinc selenide. The initial guess used in the data analysis for the refractive index of each of these materials was 4 . The initial guesses used for the thickness were those specified by the manufacturer of 4,3 , 2 , and $4 \mathrm{~mm}$, respectively. The experimentally determined thicknesses for each are displayed in Table 2. Overall, the measured refractive indices for the four materials agree fairly well with the reported or predicted values. ${ }^{9,10}$ Thickness measurements at room temperature for the sample materials agree with direct physical measurements made with a highprecision micrometer and a laser micrometer, whose uncertainties are \pm 5 and $\pm 2.5 \mu \mathrm{m}$, respectively.
Table 2. Temperature-Dependent Thickness Results for GaAs, Ge, Si, and $\mathrm{ZnSe}$

\begin{tabular}{lccc}
\hline Material & $\begin{array}{c}\text { Temperature } \\
(\mathrm{K})\end{array}$ & $d$, Measured $(\mathrm{mm})$ & $\begin{array}{c}d, \text { Vendor } \\
(\mathrm{mm})\end{array}$ \\
\hline $\mathrm{GaAs}$ & 296 & $4.0351 \pm 0.0087$ & 4 \\
$\mathrm{Ge}$ & 296 & $2.9606 \pm 0.007$ & 3 \\
& 93 & $2.9551 \pm 0.0018$ & 3 \\
$\mathrm{Si}$ & 296 & $2.0332 \pm 0.0014$ & 2 \\
$\mathrm{ZnSe}$ & 296 & $4.0418 \pm 0.0026$ & 4 \\
& 97 & $4.0376 \pm 0.0017$ & 4 \\
\hline
\end{tabular}

The initial guess used for each measurement was the vendorspecified thickness.

Theoretically, the precision of this method is dependent on the precision of the angle measurement and the stability and accuracy of the wavelength of light being used, according to Eqs. (2), (4), and (5). The sample angle is recorded from the computercontrolled precision rotation stage with a fractional resolution of $10^{-4}$ to $10^{-5}$. The fractional uncertainty of the laser wavelength is of the order of $10^{-5}$. Experimentally though, the largest uncertainty observed is the reproducibility of the measured value and is typically of the order of $10^{-3}$ to $10^{-4}$. The experimental precision is limited by a variety of factors including sample surface quality, vibrations in any of the optical components, temperature drifts in the ambient air, air currents, laser mode, and power stability. Optically flat and parallel surfaces are desired as any surface imperfections scatter the light and add phase distortions to the sample beam. Small variations in the position-dependent sample thickness and surface parallelism can also have adverse effects on the accuracy and repeatability of the measurements. The reproducibility uncertainties for each of the experimental conditions are reported in Tables 1 and 2 and are typically in the third decimal place.

Out of the four materials, germanium and zinc selenide were chosen for cryogenic measurements owing to their comparable thermal-expansion coefficients, ${ }^{11,12} 5.8 \times 10^{-6} \mathrm{~K}^{-1}$ and $7.7 \times 10^{-6} \mathrm{~K}^{-1}$, and quite different thermo-optic coefficients, ${ }^{9} \mathrm{~d} n / \mathrm{d} T$, $4.1 \times 10^{-4} \mathrm{~K}^{-1}$ and $6 \times 10^{-5} \mathrm{~K}^{-1}$ at room temperature, respectively. With the data from Table 2, the average thermo-optic coefficients, $\Delta n / \Delta T$, for Ge and ZnSe can be calculated between room temperature and cryogenic temperatures from our experimental results and are displayed in Table 3, along with calculated values by use of Hawkins and Hunneman's models. ${ }^{9}$

Table 3. Calculated Average Thermo-Optic Coefficients for Ge and ZnSe over the Temperature Ranges of 93 to $296 \mathrm{~K}$ and 97 to $296 \mathrm{~K}$, Respectively

\begin{tabular}{ccc}
\hline Material & $\begin{array}{c}\text { Average } \Delta n / \Delta T, \\
\text { Measured }\left(K^{-1}\right)\end{array}$ & $\begin{array}{c}\text { Average } \Delta n / \Delta T, \\
\text { Calculated }^{9}\left(K^{-1}\right)\end{array}$ \\
\hline $\mathrm{Ge}$ & $(3.5 \pm 0.2) \times 10^{-4}$ & $4.1 \times 10^{-4}$ \\
$\mathrm{ZnSe}$ & $(5.5 \pm 2.1) \times 10^{-5}$ & $6 \times 10^{-5}$ \\
\hline
\end{tabular}


In conclusion, we have demonstrated that Michelson and Fabry-Perot interferometry can be used sequentially to accurately measure absolute and independent values for the refractive index and physical thickness for optical materials having flat and parallel surfaces. With this technique, measurements can be made for samples under experimental conditions and temperatures in which both $n$ and $d$ are changing and in which direct physical measurements of the material thickness is difficult. The method is sensitive enough to measure refractive indices with uncertainties in the third decimal place, thickness measurements to within $\pm 5 \mu \mathrm{m}$, and average thermo-optic coefficients to within $15 \%$ of their expected values, without accurate prior knowledge of either $n$ or $d$. The method is important for practical applications in which accurate values of refractive indices of materials available only in thin wafer form are needed over a range of temperatures. Extension of this research for refractive-index measurements by use of any other desired fixed-wavelength laser source should be straightforward.

\section{References}

1. C. A. Proctor, "Index of refraction and dispersion with the interferometer," Phys. Rev. 24, 195-201 (1907).

2. M. S. Shumate, "Interferometric measurement of large indices of refraction," Appl. Opt. 5, 327-331 (1966).
3. U. Schlarb and K. Betzler, "Influence of the defect structure on the refractive indices of undoped and Mg-doped lithium niobate," Phys. Rev. B 50, 751-757 (1994).

4. J. F. H. Nicholls, B. Henderson, and B. H. T. Chai, "Accurate determination of the indices of refraction of nonlinear optical crystals," Appl. Opt. 36, 8587-8594 (1997).

5. J. C. Brasunas and G. M. Curshman, "Interferometric but nonspectroscopic technique for measuring the thickness of a transparent plate," Opt. Eng. 34, 2126-2130 (1995).

6. G. Coppala, P. Ferraro, M. Iodice, and S. De Nicola, "Method for measuring the refractive index and the thickness of transparent plates with a lateral-shear, wavelength-scanning interferometer," Appl. Opt. 42, 3882-3887 (2003).

7. M. Born and E. Wolf, Principles of Optics, 7th ed. (Cambridge U. Press, Cambridge, UK, 2002).

8. G. D. Gillen and S. Guha, "Refractive-index measurements of zinc germanium diphosphide at 300 and $77 \mathrm{~K}$ by use of a modified Michelson interferometer," Appl. Opt. 43, 2054-2058 (2004).

9. G. Hawkins and R. Hunneman, "The temperature-dependent spectral properties of filter substrate materials in the farinfrared (6-40 $\mu \mathrm{m})$," Infrared Phys. Technol. 45, 69-79 (2004).

10. E. D. Palik, Handbook of Optical Constants of Solids (Academic, New York, 1998).

11. I. S. Grigoriev and E. Z. Meilikhov, Handbook of Physical Quantities (CRC Press, Boca Raton, Fla., 1997).

12. V. Kumar and B. S. R. Sastry, "Thermal expansion coefficient of binary semiconductors," Cryst. Res. Technol. 36, 565-569 (2001). 